\title{
ENTRE LA TDT Y EL ORDENADOR: NUEVAS TENDENCIAS \\ TECNOLÓGICAS, EMPRESARIALES Y DE CONSUMO ALREDEDOR DE LA FICCIÓN AUDIOVISUAL EN ESPAÑA ${ }^{1}$
}

\section{TDT BETWEEN AND THE COMPUTER: TECHNOLOGY TRENDS, CONSUMER AND BUSINESS AROUND THE AUDIOVISUAL FICTION IN SPAIN}

\begin{abstract}
AUTOR
Luís Fernando Morales Morante: Investigador Postdoctoral y profesor del Departamento de Comunicación Audiovisual y Publicidad II, Universidad Autónoma de Barcelona (España). fernando.morales@uab.es
\end{abstract}

\section{RESUMEN}

Las dinámicas distributivas y de consumo audiovisual han sufrido recientemente cambios importantes provocados por la convergencia mediática e Internet como eje transversal insustituible para la transmisión de señales. Un sector donde se percibe con particular nitidez estos cambios en la comunicación es la ficción audiovisual. En el presente artículo se realiza un estudio de los componentes tecnológicos, políticos y económicos que inciden actualmente la creación y circulación de productos de ficción en España. Los resultados indican tres cuestiones importantes: primero: la estandarización de Internet como mecanismo de transmisión audiovisual multiplataforma; segundo: la inexistencia de una industria de la ficción sólida, incapaz de acercarse a nuevos mercados y tercero: la falta de políticas reguladoras oportunas para ordenar eficazmente el funcionamiento de las dinámicas actuales de la producción y el mercado.

\footnotetext{
${ }^{1}$ La presente investigación forma parte del proyecto Convergtvd: Producción, economía, contenidos y públicos de la ficción televisiva multipantalla. Financiado por el Ministerio de Ciencia e Innovación, de España, CS02009-12568-C03-01.
} 


\section{PALABRAS CLAVE}

Ficción audiovisual - Producción - Televisión - Internet - Multiplataforma

\section{ABSTRACT}

Distributional dynamics and audiovisual consumption have recently been significant changes caused by media convergence and the Internet as a crosscutting indispensable for the transmission of signals. One area which is seen with particular clarity in communicating these changes is the audiovisual fiction. The present article is a study of the technological components, political and economic factors that currently affect the creation and movement of fiction in Spain. The results indicate three important issues: first, the standardization of the Internet as platform audiovisual transmission mechanism, second: the lack of a solid fiction industry, unable to approach new markets and third, the absense of appropriate regulatory policies to effectively manage des operation current dynamics of production and market.

\section{KEY WORDS}

Audiovisual fiction film - Production - Television - Internet - Platform

\section{ÍNDICE}

1. PRESENTACIÓN

2. CONTEXTO AUDIOVISUAL ESPAÑOL VARIABLES TECNOLÓGICAS ACTUALES

3. CONGLOMERADOS ECONÓMICOS DEL AUDIOVISUAL

4. EVOLUCIÓN DE LAS PRÁCTICAS DE ACCESO A LOS CONTENIDOS DE TV

4.1. Indicadores en las nuevas plataformas de consumo

5. LA DISTRIBUCIÓN DE AUDIENCIAS 
5.1. La cuota de ficción en las pantallas

5.2. Nexos entre productoras y cadenas de TDT

5.3. La descarga y visionado de productos ficcionales

6. DISCUSIÓN

6.1. La tecnología

6.2. Consumo

6.3. La dinámica empresarial y regulación del estado

7. CONCLUSIONES Y PERSPECTIVAS FUTURAS

8. REFERENCIAS

\section{PRESENTACIÓN}

En la presente década hemos podido presenciar un conjunto de cambios estructurales profundos en el sistema televisivo por la evolución trepidante de las tecnologías de transmisión de señales audiovisuales. El desarrollo y penetración de Internet como plataforma básica para la transportación de datos ha terminado instaurándose en el funcionamiento de otros protocolos y formas accesibles para la difusión de imagen y sonido sincrónicos: señal en vivo y grabada en diferentes formatos y compresiones digitales por ordenador. Bajo estas condiciones el modelo convencional de acceso lineal y dependiente, propio de la televisión analógica queda desplazado por un protocolo estandarizado regido por los cánones de la interactividad. Consiguientemente, la cadena de valor de la producción y distribución audiovisual se ve afectada, planteando, a partir de las posibilidades tecnológicas disponibles, nuevos mecanismos, productos y estrategias comunicativas pensadas en términos de estimular el consumo individual, participativo, especializado y fragmentado de los contenidos.

Concretamente en España, una implicación directa del boom tecnológico ha sido la manera como las empresas han aprovechado la transición digital como una oportunidad para afrontar conjuntamente las incertidumbres que el propio cambio tecnológico plantea con sus continuas innovaciones. La circunstancia de crisis ha 
aumentado aún más las dudas generales y ha contado con el aval del Estado para tratar de implantar algunas acciones concretas orientadas a reducir el riesgo de las inversiones e imponer una suerte de política directriz (correcta o no) para afrontar el futuro inmediato.

Uno de los ámbitos que ha sabido adaptarse mejor a este nuevo modelo de producción, distribución y consumo es el correspondiente a la producción de ficción audiovisual. Desde el punto de vista económico empresarial, se viene observando recientemente juntamente con las grandes alianzas estratégicas, el incremento de coproducciones entre canales y productoras, habitualmente dedicadas a crear este tipo de programas. Tres razones fuerzan este tipo de acuerdos comerciales y operación. La primera para explorar nuevas vías creativas para desarrollo de contenidos y su difusión en la nueva dinámica digital. Segundo, para compartir gastos de inversión en equipamiento de punta y asumir con más confianza los retos innovadores suscitados por la crisis. Y tercero, para explorar de manera conjunta figuras de promoción y exportación de sus productos.

Por sus características nativas el discurso de ficción ostenta distintas ventajas que lo hacen particularmente atractivo para capitalizar satisfactoriamente las nuevas ventajas del consumo televisivo en el entorno digital. La ficción puede emitirse por la TDT o por dispositivos personales. Se adapta bien a un consumo individual o grupal. Posee un formato serial y desarrolla relatos atemporales que facilitan su ajuste a distintos mercados y públicos. Es decir, permite acoplar paralelamente a su dinámica convencional nuevas fórmulas para efectuar el visionado de los episodios o establecer mecanismos de interacción innovadores, a partir de los cuales se genere mayor empatía con los destinatarios, independientemente de su localización exacta. Así, en el punto intermedio de una transición continuada, el consumo de la ficción discurre entre dos pantallas: la convencional del televisor y las nuevas pantallas: ordenador, móviles y dispositivos portátiles. 


\section{CONTEXTO AUDIOVISUAL ESPAÑOL-VARIABLES TECNOLÓGICAS ACTUALES}

Hoy en día Internet constituye un medio indispensable para el funcionamiento de cualquier tecnología de difusión o para generar interacciones amigables entre diferentes protocolos como la IPTV, HD, P2P (Bikfalvi, García-Reinoso, Vidal \& Valera: 2009) e inclusive en los más recientes experimentos de televisión en tres dimensiones.

Si bien el principal evento mediático de la televisión reciente en España ha sido el fin del sistema de transmisión analógico y la homogenización europea con la TDT, hoy se habla fundamentalmente de sus limitaciones y falta de capacidad para competir con otros modelos de teledifusión (Bustamante: 2008). Por ende, hay razones para diagnosticarle una corta vida, máxime con el surgimiento de la HD o Alta Definición. Vivimos en un proceso de múltiples nacimientos pero pocas consolidaciones de tecnologías, modelos, plataformas y sistemas. Este devenir hace del mapa radiodifusor un punto concéntrico donde coexisten diferentes sistemas que luchan tenazmente por instaurarse en el mercado como el modelo estándar del futuro en Europa (Reading: 2008). Juntamente con el Apagón analógico, emerge la TDTHD como modelo de mayor calidad y normas técnicas diferentes. Pese a ello la TDTHD se transmite haciendo uso de la misma plataforma de TDT convencional, incluso los receptores estándar pueden verla mediante un televisor con entrada HD o un decoder compatible. En medio de estos giros surgen los primeros experimentos serios de emitir en 3D. En Catalunya vienen haciéndose transmisiones en prueba desde el 2008 y probablemente en dos años se haga el lanzamiento oficial del sistema, al tiempo que la empresa SONY comienza a vender sus nuevos modelos de televisor con conexión a Internet compatibles con el formato 3D. No obstante, otras compañías están desarrollando sus propios receptores y se avizora en breve plazo una competencia dura para lograr implantarse en el mercado y es un hecho que la oferta actual será mejorada en prestaciones y flexibilidad.

Paralelamente, el sector de la televisión IP (IPTV) sigue controlando la mayor franja del mercado junto con los operadores de cable y satélite (Delgado \& Fernández: 2006). En 
medio de las dificultades por mejorar el volumen de suscriptores, el sector ha logrado incrementar sus utilidades netas respecto de años anteriores. La innovación tecnológica también fuerza las mejoras en la calidad de la señal de emisión. El canal autonómico catalán TV3 cumple su segundo año de pruebas en Alta Definición, desde 2009 lo hace Televisión Española y recién el 2010, luego de las fusiones y redistribución de frecuencias lo inician Telecinco, Antena 3 y La Sexta. Pero el mapa no se acaba de trazar sin la finalización del proceso de adaptación de antenas y plantas retransmisoras de alcance nacional iniciado el 2005. Actualmente, tras la instalación de estaciones en los focos y poblados desatendidos, se alcanza una cobertura efectiva del $98,36 \%$ del territorio ${ }^{2}$. Los avances hacia la televisión 3D aún son tímidos, por lo pronto solo podemos mencionar las emisiones de prueba en 3D que viene realizando la televisora pública TV3. Finalmente, tenemos al negocio de la televisión digital de pago en sus distintas plataformas. Con ofertas temáticas variadas, intentan aliarse con otros operadores para aumentar su competitividad y presencia, negociando por separado la señal de algunos de sus canales más populares (Telefónica-GolTV, Canal+Ono).

\section{CONGLOMERADOS ECONÓMICOS DEL AUDIOVISUAL}

Además de la innovación tecnológica, en los últimos dos años la televisión digital en España ha experimentado algunos cambios significativos desde el punto de vista empresarial y la organización de su propuesta programática. Un primer factor consiste en la consolidación de las cadenas generalistas que durante el último año de emisiones analógicas ya venían operando en norma digital. Los pasos para facilitar el ingreso hacia este nuevo modelo eran sólidos e incluía una adaptación bastante ágil de los usuarios, animados con los cambios. Desde el año 2006 se registra un incremento sostenido de la oferta de televisores con receptores de TDT incorporados y decodificadores para adaptar rápidamente los aparatos antiguos ante la inminente extinción de las emisiones analógicas. Las ventas de receptores en los cuatro últimos años continuaron en aumento. El informe estadístico de Impulsa TV correspondiente al 
mes de marzo del 2010 reporta una cifra total superior a 28 millones de descodificadores de TDT vendidos en toda España. El doble respecto de la cifra del 20083. También, las ventas de televisores con TDT incorporado se elevan superando las 500 mil unidades, mientras van apareciendo los nuevos televisores con sintonizador HD y nuevos puertos para su fácil conexión con otros dispositivos.

En el último año las políticas públicas han contribuido en la consolidación incentivando la formación de alianzas estratégicas empresariales vinculados al sector del audiovisual. Concretamente el Estado, en aparente previsión de los efectos que podía provocar la crisis internacional en este sector, impulsa un conjunto de medidas urgentes para favorecer la fusión del mercado y liberalizarlo en el momento crucial de la transición digital. La norma más importante en este sentido es la Ley 7/2009 de Medidas Urgentes en Materia de Telecomunicaciones ${ }^{4}$, y modifica el decreto que suprime el tope del 5\% de participaciones cruzadas entre accionistas de las cadenas. Esta disposición permite a los grupos existentes tener acciones hasta en dos cadenas, siempre que no superen entre ambas el $27 \%$ de la audiencia total nacional. Con esta medida, cualquier combinación inter empresarial era posible, a excepción de una fusión entre Gestevisión Telecinco y Antena 3. Pero en un principio fueron mayores los temores que el embate de la crisis y no fue hasta el último trimestre del 2009, cuando comenzaron a aparecer informaciones fiables sobre posibles acuerdos. Finalmente, fue el 18 de diciembre de 2009, cuando PRISA y Mediaset, accionistas mayoritarias de Sogecable y Gestevisión Telecinco, respectivamente, presentaron un acuerdo de fusión para sus cadenas de televisión en abierto. Tras esta alianza, Cuatro y su licencia de emisión se separaron de Sogecable, y esta última fue comprada, en su totalidad por Gestevisión Telecinco. Junto con este acto, PRISA compró acciones de nueva emisión de Gestevisión Telecinco, equivalentes al 18\% de su capital social. Con estas reformas, el mapa empresarial de la TDT queda constituido finalmente por seis grupos privados: Antena 3, Gestevisión-Telecinco, Cuatro, 
Gia La Sexta, Veo Televisión y Sociedad gestora de televisión; y el grupo de TVE en calidad de servicio público en exclusiva (ver Tabla 1).

\begin{tabular}{|l|c|c|}
\hline Radio Televisión Española & La 2, 24h, Teledeporte, Clan & TVE HD \\
\hline Antena 3 de Televisión & Antena 3, Neox, Nova, Nitro & Antena 3 HD \\
\hline $\begin{array}{l}\text { Gestevisión Telecinco } \\
\text { Cuatro }\end{array}$ & $\begin{array}{c}\text { Telecinco, LaSiete, } \\
\text { Factoría de Ficción, Boing } \\
\text { GH 24 horas Canal+ Dos }\end{array}$ & Telecinco HD \\
\hline $\begin{array}{l}\text { Gestora de Inversiones } \\
\text { Audiovisuales La Sexta }\end{array}$ & $\begin{array}{r}\text { laSexta, laSexta2, laSexta3, } \\
\text { Gol Televisión }\end{array}$ & laSexta HD \\
\hline Veo Televisión & \multicolumn{2}{|c|}{ Veo7, 13 TV } \\
Sociedad Gestora de \\
Televisión
\end{tabular}

Tabla 1: Distribución de canales de TDT de cobertura nacional según grupos empresariales

El esquema resulta bastante ilustrativo porque refleja la distribución de las cadenas. Deja observar la segmentación y la orientación respecto de la línea temática dominante para cada una. Como puede apreciarse, los canales que actualmente se dedican a difundir íntegramente contenidos de ficción son: por Gestevisión-Cuatro: FDF, La Siete, Boing; por el grupo Antena 3: Neox y Nova; por el grupo Veo lo hace: AXN; por Net, Disney Channel. La suma del conjunto de canales equivale entonces a un $47,6 \%$ de la audiencia de las cadenas temáticas.

\section{EVOLUCIÓN DE LAS PRÁCTICAS DE ACCESO A LOS CONTENIDOS DE TV}

Las formas tradicionales de relacionarnos con los medios, aparatos y contenidos han sufrido también transformaciones inducidas por la revolución tecnológica. La variedad de opciones y calidades para visionar y registrar señales audiovisuales de la televisión vía satélite y los receptores digitales, ha incrementado nuestra destreza instrumental, convirtiéndonos en espectadores expertos «proactivos» para seleccionar interactivamente la información de un inmenso repositorio que va creciendo y alimentándose diariamente de diferentes fuentes: desde materiales generados por las grandes productoras y distribuidoras hasta de jóvenes novatos en la creación audiovisual. El crecimiento y la capacidad de cobertura de Internet han solidificado el uso de pantallas alternativas a la televisión, reflejando un decrecimiento sistemático de las a11diencias a11e han miorado al florecimiento a11diovisılal del ordenador. I on ióvenes 
dedican gran cantidad de horas al visionado y descarga de episodios íntegros de programas. Pero advertimos un aspecto negativo en medio de este natural incremento de la ofertas. La proliferación de medios y pantallas de visionado atomiza todavía más las audiencias, porque dificulta la localización certera de la información, aún cuando los buscadores orienten y depuren por determinados patrones de búsqueda. Es tan amplio entonces el volumen de canales, programas, temáticas que se copian, editan y difunden por diferentes medios y plataformas en tan poco tiempo, que pueden resultar inadvertidos para la audiencia potencial $\mathrm{u}$ otros sectores interesados. La carrera por dar a conocer descolladamente las innovaciones parece producirse cada vez más rápidamente. Se gana en interacción, funciones y mensajes personalizados que se pierde en el tiempo y son sustituidos por otros mejores en sus prestaciones y calidad. El cambio de las prácticas de consumo y la ampliación hacia un uso cada vez más maleable para entrar en contacto con otros medios y plataformas, marca el valor del avance tecnológico de la televisión en el transcurso del tiempo (ver Tabla 2).

\begin{tabular}{|c|c|c|c|c|}
\hline & FASES & $\begin{array}{l}\text { AUDIENCIA/ } \\
\text { CONSUMO }\end{array}$ & $\begin{array}{l}\text { INTERACCIÓN } \\
\text { RECEPTOR }\end{array}$ & $\begin{array}{l}\text { INTERACCIÓN } \\
\text { OTROS MEDIOS }\end{array}$ \\
\hline 1 & $\begin{array}{c}\text { TV } \\
\text { Analógica }\end{array}$ & $\begin{array}{l}\text { Heterogénea } \\
\text { Total } \\
\text { Pasiva }\end{array}$ & $\begin{array}{l}\text { Visionado lineal } \\
\text { Elección simple de la } \\
\text { oferta limitada } \\
\text { Sin interacción }\end{array}$ & Nula \\
\hline 2 & $\begin{array}{l}\text { TV } \\
\text { Color y } \\
\text { Cable }\end{array}$ & $\begin{array}{l}\text { Heterogénea } \\
\text { Segmentada } \\
\text { Activa para la } \\
\text { búsqueda de } \\
\text { contenidos }\end{array}$ & $\begin{array}{l}\text { Visionado lineal } \\
\text { Elección simple de una } \\
\text { oferta amplia de canales } \\
\text { Sin interacción }\end{array}$ & $\begin{array}{c}\text { Estaciones de Radio } \\
\text { Teletexto } \\
\text { Servicios de pago } \\
\text { Premium }\end{array}$ \\
\hline 3 & $\begin{array}{l}\text { Neo } \\
\text { TV }\end{array}$ & $\begin{array}{c}\text { Individual } \\
\text { Atomizada } \\
\text { Activa y migratoria } \\
\text { con otros medios y } \\
\text { soportes }\end{array}$ & $\begin{array}{c}\text { Visionado lineal y } \\
\text { no lineal } \\
\text { A la carta (pago) } \\
\text { En cualquier momento y } \\
\text { lugar }\end{array}$ & $\begin{array}{c}\text { Internet, IPTV, P2P } \\
\text { Streaming, Cross media } \\
\text { Digital, TV, Descargas y } \\
\text { visionado en Móviles y } \\
\text { Portátiles }\end{array}$ \\
\hline
\end{tabular}

Tabla 2: Evolución de las prácticas en la interacción con las tecnologías audiovisuales. Creación propia

En una primera fase, anterior a 1980, ver la televisión constituía una práctica familiar de consumo pasivo, donde la televisora construía una programación generalista para un público homogéneo. El espectador recibía los contenidos de forma unidireccional y simplemente elegía de una oferta limitada de canales y programas. 
Entre los años 1990 al 2000 se producen cambios importantes e ingresamos a una fase dominada por el crecimiento de la oferta de canales y contenidos que ingresan además por las señales de satélite y el cable. En esta etapa aparece el primer servicio añadido: el Teletexto. Comienzan las primeras emisiones codificadas previo pago y la recepción de señales de radio a través de Internet.

La fase actual se inicia el año 2000 y está caracterizada por un nuevo incremento de la oferta multicanal. El nacimiento y consolidación de prácticas interactivas hombremáquina con una amplia gama de dispositivos para acceder a la información. El actual proceso de fusión entre Internet y Televisión encarnado por la IPTV y sus variantes, ha servido para generar nuevas maneras para relacionarnos con los contenidos. Ahora se accede desde dispositivos portátiles iPad, Smarthphones, y teléfonos móviles 4G, basadas íntegramente en el intercambio de datos por IP: Blackberry o SciPhone, con aplicaciones multimedia, agenda y aplicativos para todos los perfiles de usuarios.

\subsection{Indicadores en las nuevas plataformas de consumo}

Los principales equipos para la conexión a Internet siguen siendo los ordenadores personales. Los de sobremesa en un $87 \%$ y los portátiles son los más utilizados para conexiones a la Red. Se registra un aumento de accesos desde teléfonos móviles del $32,7 \%$, ocupando la tercera posición. En la escala de preferencias le sigue en un cuarto lugar la videoconsola con 17,1 y las Agendas PDA con 9,4\%. La suma de porcentajes excede el 100\% debido a que los usuarios acceden a varios dispositivos simultáneamente. Los datos que se reportan (ver Tabla 5) retratan ciertamente una «fotografía instantánea» de la evolución de la conectividad al 2009 y ponen en evidencia el incremento del uso de móviles y PDAS en la práctica de descarga de contenidos audiovisuales de ficción. Cifra que es mayor respecto de los años previos. Muy probablemente dicha tendencia continúe en aumento por dos factores de desarrollo tecnológico. Por un lado, debido al auge del Netbook, la salida al mercado del Ipad y otros dispositivos para el visionado on line y descarga de materiales audiovisuales. Por 
otra parte, por la salida en el mercado de televisores táctiles con acceso directo a Internet en varias marcas. Consideramos que ambas tecnologías modificarán severamente las formas del consumo, trasladándolas al uso masivo de pantallas amplias. Finalmente, se producirá un encuentro donde los contenidos serán vistos con la misma calidad tanto en ordenadores como televisores, con la ventaja de los primeros sirven para acceder de forma instantánea a archivos alojados en servidores, sin depender de la forma tradicional del acceso o formas de interactividad que ofrecen los diferentes decodificadores de televisión digital.

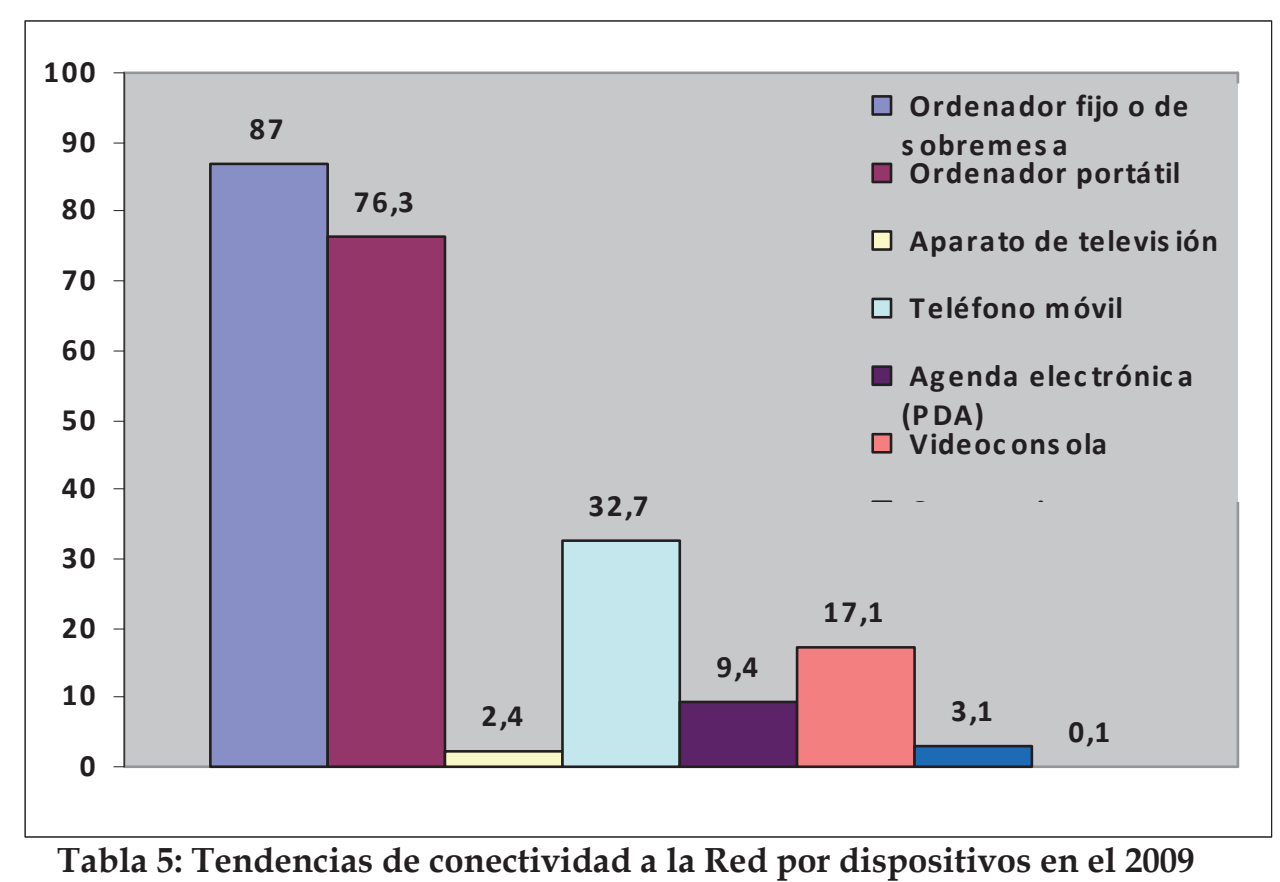

Fuente: EGM-AIMC

\section{LA DISTRIBUCIÓN DE AUDIENCIAS}

Independientemente de los condicionantes económicos de carácter general y la migración de la teleaudiencia hacia pantallas alternativas de recepción, la aparición de nuevos canales temáticos ha contribuido a la formación de la actual oferta programática fragmentada. Sin duda, este hecho representa un fenómeno nuevo, propio de la TDT, en tanto que la emisión de un mayor número de canales por operador solo es posible porque el sistema permite la multiplexación o subdivisión de la frecuencia asignada por el ente estatal en múltiples canales para destinarlos a la emisión de múltiples señales: televisión. radio v si es necesario codificarlos para ofertarlos previo pago. Analizando la 
repartición de las audiencias por canales y grupos empresariales en 2010 en España (ver Gráfico 1), vemos que la mayor cuota de pantalla sigue conservándola la corporación estatal RTVE con un 47,1\%, seguido por el Grupo Antena 3 con 45,4, Gestevisión con 31,5, y por debajo, se encuentran NetTV con 13,9\%, Grupo Veo con 11,7\% y finalmente, Gestora de Inversiones Audiovisuales con solo 9,7\%. La cifra total incluye tanto los canales en abierto generalistas como las televisiones temáticas.

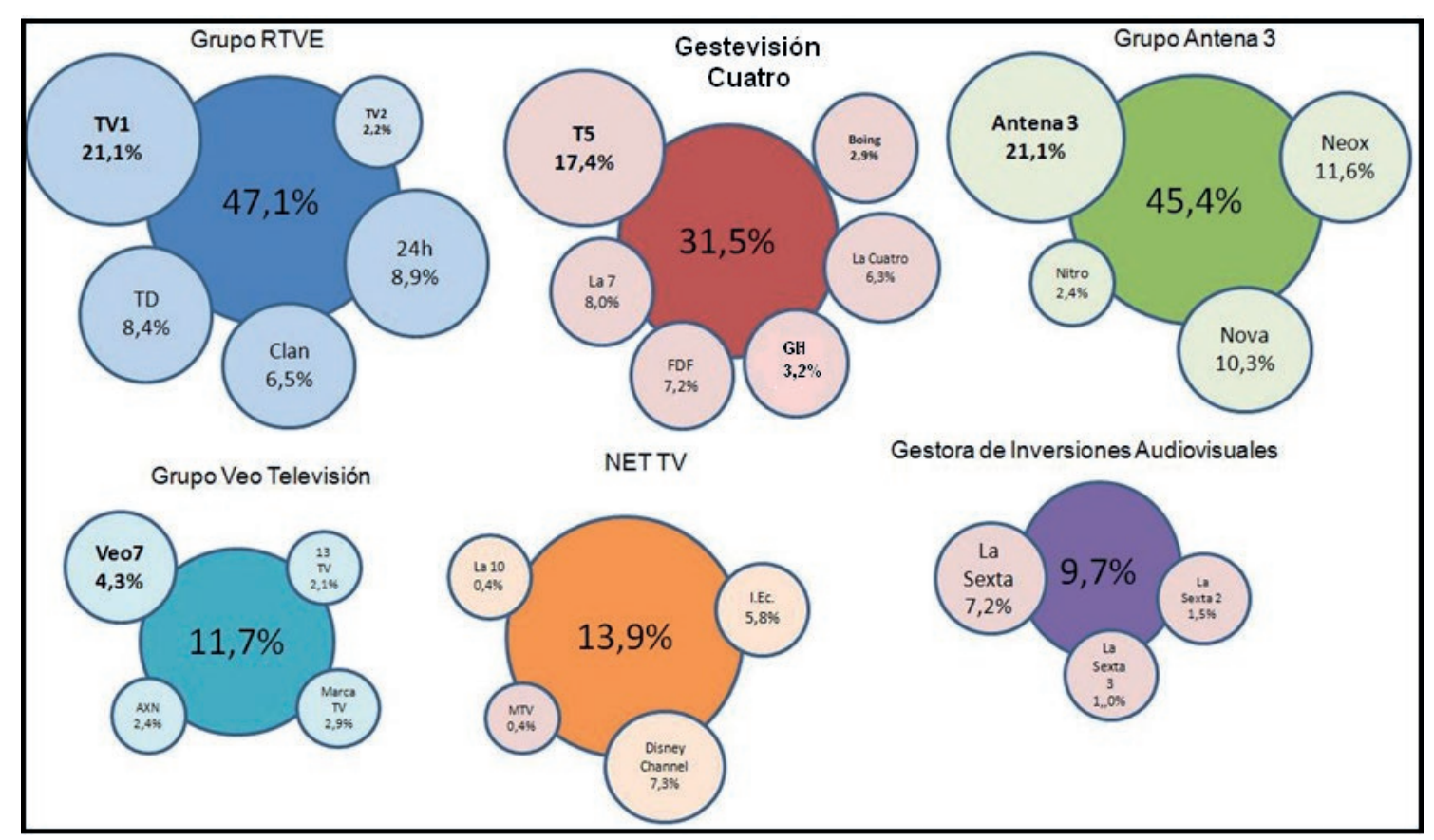

Gráfico 1: Distribución de audiencias en el nuevo mapa de canales TDT

\subsection{La cuota de ficción en las pantallas}

Si dirigimos nuestra atención concretamente hacia los canales temáticos (ver Tabla 3), comprobamos el liderazgo de Antena Neox y Antena Nova, señales alternas del Grupo Antena 3. Juntamente alcanzan una audiencia de 19,85\% del total. Ambas cadenas poseen una marcada preferencia por productos de ficción de importación, principalmente telenovelas de procedencia latinoamericana. Factoría de Ficción (FDF) del Grupo Telecinco, dedicada a la programación mayoritaria de series norteamericanas y europeas, se sitúa varios puestos por debajo con una cuota de 5,7\%. El resto de canales se dedican a ofertas no ficcionales: informativos, música, deportes, televentas, entre 
otros, poseen índices mínimos. La estadística de EGM-AIMC ${ }^{5}$ expresa el porcentaje medio mensual para cada canal, al mes de marzo del 2010.

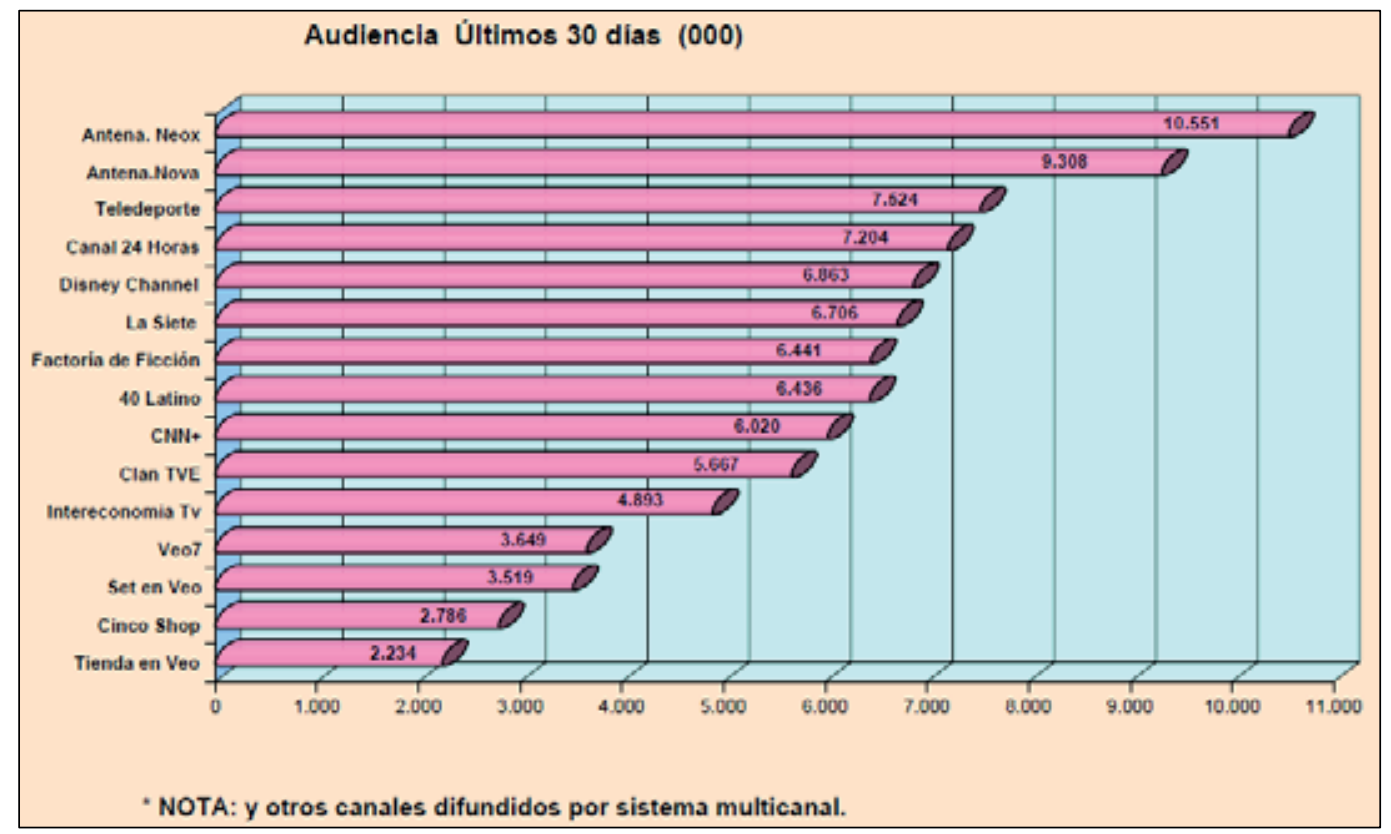

Tabla 3: Audiencia de canales temáticos de cobertura nacional a Marzo de 2010 Fuente: EGM-AIMC

\subsection{Nexos entre productoras y cadenas de TDT}

¿Pero cómo se articulan las relaciones entre las empresas de producción y los canales generalistas de TDT? El mapa conceptual siguiente muestra el volumen de inserción de contenidos de ficción en los canales españoles. La empresa Globomedia es la que goza de mayor presencia mediática al exhibir sus programas en un total de 5 canales: Antena 3, Telecinco, La Sexta, TVE y el canal autonómico del País Vasco ETB1. Boomerang lo hace en TVE, Antena 3 y Telecinco. Diagonal también emite en tres canales: Antena 3, TVE y la cadena autonómica de Cataluña TV3. Finalmente, con dos cadenas tenemos a Notro y Zepellin. Con una queda Pausoka que emite exclusivamente para el canal local ETB1 en el País Vasco. 


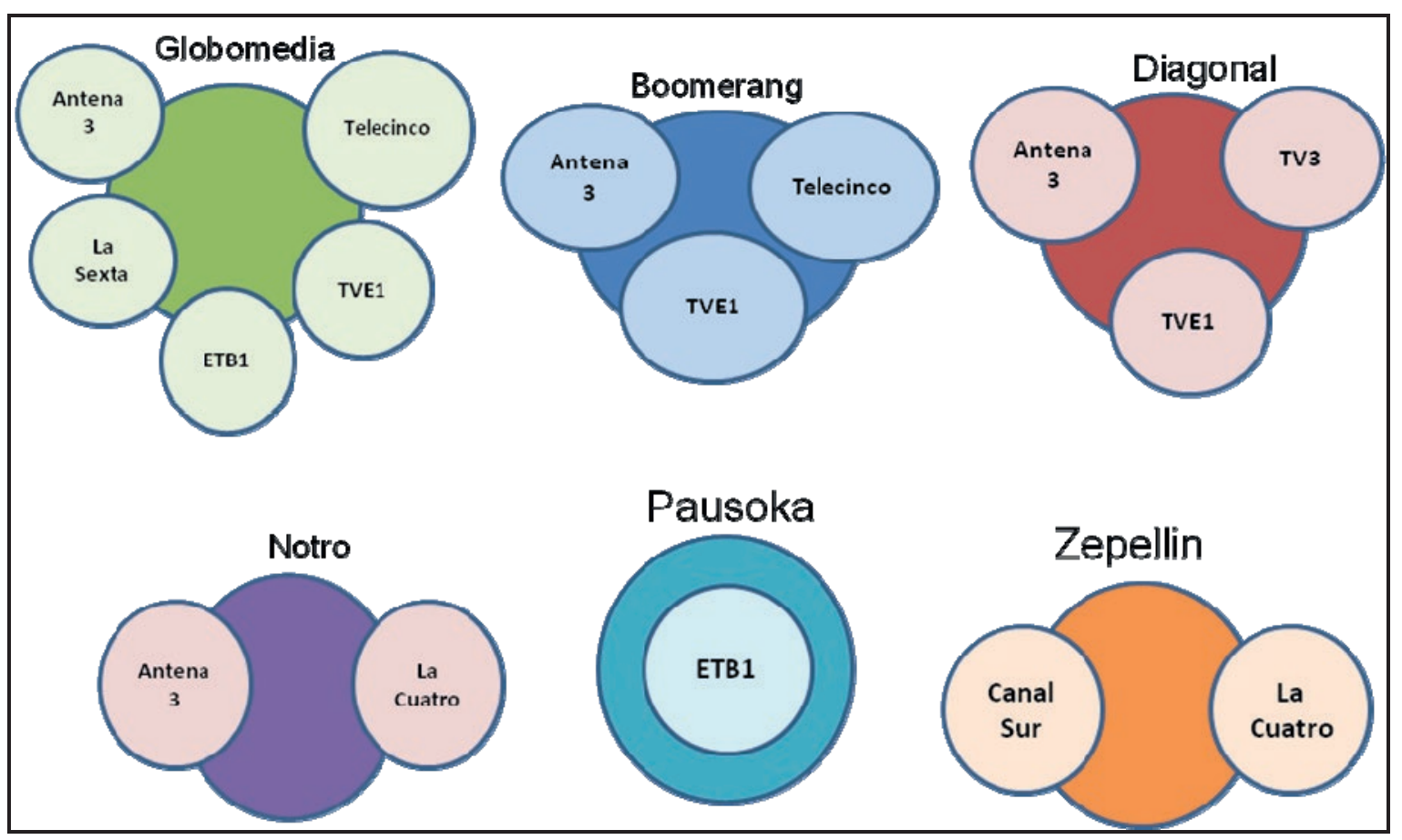

Gráfico 2: Mapa de inserción de contenidos de las productoras en cadenas nacionales Creación propia

Seguidamente analicemos brevemente el resultado de cada uno de los programas producidos.

Se observa claramente que Globomedia prefiere desarrollar sus proyectos de forma individual. De los cinco programas producidos Águila Roja (TVE1), Aída (T5), El Internado y Los Hombres de Paco (A3) y La Tira (La Sexta), solo este último se ubica en el horario de la tarde y es justamente el de más baja audiencia (6,0). Los restantes tienen entre 16,5: Los Hombres de Paco (A3) y 24,3\% de share: Águila Roja (TVE1). Se produjeron solo dos coproducciones una con otra productora vasca Tentazioa, puntualmente para producir la comedia familiar Martin. También firmó un convenio con A3Films para realizar la miniserie de dos capítulos Una bala para el Rey, cuya expectativa colectiva no se corresponde con los 13,8 alcanzados.

\begin{tabular}{|l|c|c|c|c|}
\hline Productora & Título originales & Canal & Franja & Share \\
\hline Globomedia & Águila Roja & TVE1 & 3 PT & 24,3 \\
\hline Globomedia & Aída & T5 & 3 PT & 21,6 \\
\hline Globomedia & El Internado & A3 & 3 PT & 17,2 \\
\hline Globomedia & La Tira & LA SEXTA & 2 Ta & 6,0 \\
\hline Globomedia & Los Hombres de Paco & A3 & 3 PT & 16,5 \\
\hline $\begin{array}{l}\text { Globomedia } \\
\text { \& Tantosina }\end{array}$ & Martin & ETB1 & 3 PT & 0,2
\end{tabular}




\begin{tabular}{|c|c|c|c|c|}
\hline $\begin{array}{l}\text { Globomedia } \\
\& \text { A3 Films }\end{array}$ & Una Bala para el Rey & A3 & 3 PT & 13,8 \\
\hline Boomerang & Acusados & T5 & $3 \mathrm{PT}$ & 16,0 \\
\hline Boomerang & La Chica de Ayer & A3 & $3 \mathrm{PT}$ & 14,8 \\
\hline Boomerang & Los Misterios de Laura & TVE1 & $3 \mathrm{PT}$ & 16,0 \\
\hline Diagonal & $\begin{array}{c}90-60-90 \\
\text { Diario Secreto de una Adolescente }\end{array}$ & A3 & 3 PT & 13,5 \\
\hline Diagonal & $\begin{array}{l}\text { Infidels } \\
\end{array}$ & TV3 & 3 PT & 2,5 \\
\hline Diagonal & La Señora & TVE1 & $3 \mathrm{PT}$ & 18,3 \\
\hline Diagonal & Ventdelplá & TV3 & $3 \mathrm{PT}$ & 3,1 \\
\hline Diagonal & Amar en Tiempos Revueltos & TVE1 & $2 \mathrm{Ta}$ & 22,5 \\
\hline Notro & Cuestión de Sexo & CUATRO & $3 \mathrm{PT}$ & 7,4 \\
\hline Notro & Doctor Mateo & A3 & $3 \mathrm{PT}$ & 17,0 \\
\hline Notro & HKM & CUATRO & $2 \mathrm{Ta}$ & 5,7 \\
\hline Notro & La Familia Mata & A3 & $3 \mathrm{PT}$ & 10,6 \\
\hline Pausoka & Euskolegas & ETB2 & $3 \mathrm{PT}$ & 0,8 \\
\hline Pausoka & Go!Azen & ETB1 & $3 \mathrm{PT}$ & 0,3 \\
\hline Pausoka & Goenkale & ETB1 & $3 \mathrm{PT}$ & 0,1 \\
\hline Zeppelin & Arrayán & C.SUR & $3 \mathrm{PT}$ & 3,7 \\
\hline Zeppelin & Los Exitosos Pells & CUATRO & $2 \mathrm{Ta}$ & 3,3 \\
\hline Zeppelin & Ponme una Nube Rocío & C.SUR & $3 \mathrm{PT}$ & 1,8 \\
\hline
\end{tabular}

Tabla 4: Producción nacional de ficción por productoras Fuente OITVE

Boomerang distribuye sus creaciones en tres cadenas T5 Acusados, A3 La Chica de Ayer y TVE1 Los Misterios de Laura. Los tres espacios se programan en prime time y obtienen entre 14,8 y $16 \%$ de share.

La atención de Diagonal está fijada solamente en dos cadenas TV1 y TV3. Los programas más exitosos son precisamente los transmitidos por la cadena pública TV1, de ellos Amar en tiempos revueltos puede visionarse también mediante su página Web. Las series de TV3 Vendelplá y Infidels, poseen una importante tradición en el rubro de la ficción. La primera es una telenovela que se alojó en el horario prime time y logró emitirse por cinco temporadas y 338 capítulos, exportada incluso a Bulgaria en 2008. Infidels es una serie dramática que ha dado también buenos resultados. Ambas dejan descargar los capítulos para verlos por el móvil y también los tienen colgados en sus respectivos portales. Los buenos resultados de estas producciones han contribuido al fortalecimiento de Diagonal como empresa de referencia de la ficción catalana.

Notro discurre entre Antena 3 y Cuatro. Tres de sus cuatro programas se emiten en horario estelar, con excepción de HKM la serie musical iuvenil aue se difunde en la 
franja de la tarde. Los productos con mejores resultados vienen a ser los emitidos por Antena 3. La televisora tiene a priori una franja de audiencia considerable en el prime time la cual actúa como una base ideal para el posicionamiento de muchos de sus programas. Cuestión de sexo pese a las reiteradas críticas de los sectores conservadores sigue el patrón de otros programas similares como Física o Química, por ejemplo.

Pausoka, de momento solo se aboca a fortalecer su relación comercial con el canal ETB del País Vasco con tres productos Euskolegas, Go!Azen y Goenkale. De momento no tienen planes de asociarse con otras empresas para intentar dar un salto a las televisoras nacionales, que bien puede ser positivo para ampliar su radio de cobertura. Escenario parecido ocurre con Zepellin, compañía perteneciente al Grupo Endemol. La misma que viene produciendo actualmente Gran Hermano vía Telecinco y que desde diciembre de 2010 tiene su propio canal 24 horas en TDT en sustitución de CNN+.

Tomando en cuenta los cambios permanentes que vienen aconteciendo en todos los niveles de la creación y distribución audiovisual, el sector de la ficción no está libre y menos renuente a explorar posibles acercamientos y fusiones, si estos pueden incidir en la mejora de su capacidad competitiva. Es viable, como punto de partida, la llegada de algunos cambios y propuestas novedosas en cuanto a la elaboración de contenidos. Por otra parte, habrá de seguir atentamente qué pasará en el futuro con las coproducciones. Si solo el mercado español exhibirá en este sentido las experiencias puntuales de Globomedia o el modelo se extenderá a otras empresas del sector, para así reducir presupuestos individuales y lograr penetrar con mayor eficacia en otros mercados españoles, de países de la Unión Europea o incluso Latinoamérica, cuya homogeneidad lingüística puede representar un factor atractivo para los compradores de los países de la región.

\subsection{La descarga y visionado de productos ficcionales}

Como hemos visto líneas arriba, muchas de las televisoras últimamente han decidido potencializar sus alojamientos web para facilitar el visionado de sus episodios de 
ficción, inclusive anunciando preestrenos de los capítulos. Esto supone algunos cambios en la lógica del consumo y plantea una serie de disquisiciones acerca de las formas de acceso a los videos y cómo los contenidos se encuentran cada vez más próximos a los televidentes e internautas. La inserción de enlaces y materiales multimedia como parte de la logística Internet, la interacción y la inmediatez para ver el contenido, son solo algunas de las razones que explican esta migración y la disminución de la audiencia de la televisión convencional unidireccional, que la propia TDT no ha sido capaz de romper. El último año hemos podido apreciar como la estrategia de TV1, Telecinco, Antena 3 y TV3 ha sido imitada por La Sexta y La Cuatro, para lograr compensar sus bajos índices de audiencia con descargas o visionado on line de sus programas.

Podemos visualizar en la siguiente tabla (ver Tabla 7) el ranking de los siete programas de ficción más descargados durante el 2009. Precisamente vemos que los dos programas con mayor número de descargas corresponden a series emitidas por Cuatro. En primer lugar se ubica Lost con un 29,8\%, luego House con 15,1\%, más relegado se encuentra la serie emitida por Antena 3 The Big Bang theory con 7,7\% y producida por la cadena norteamericana Warner. Luego encontramos la serie de La Sexta Como conocí a vuestra madre con 7,5\%. Finalmente, ubicamos a CSI de Telecinco con un 5,7\% y Flashforward de La Cuatro con $5,4 \%$.

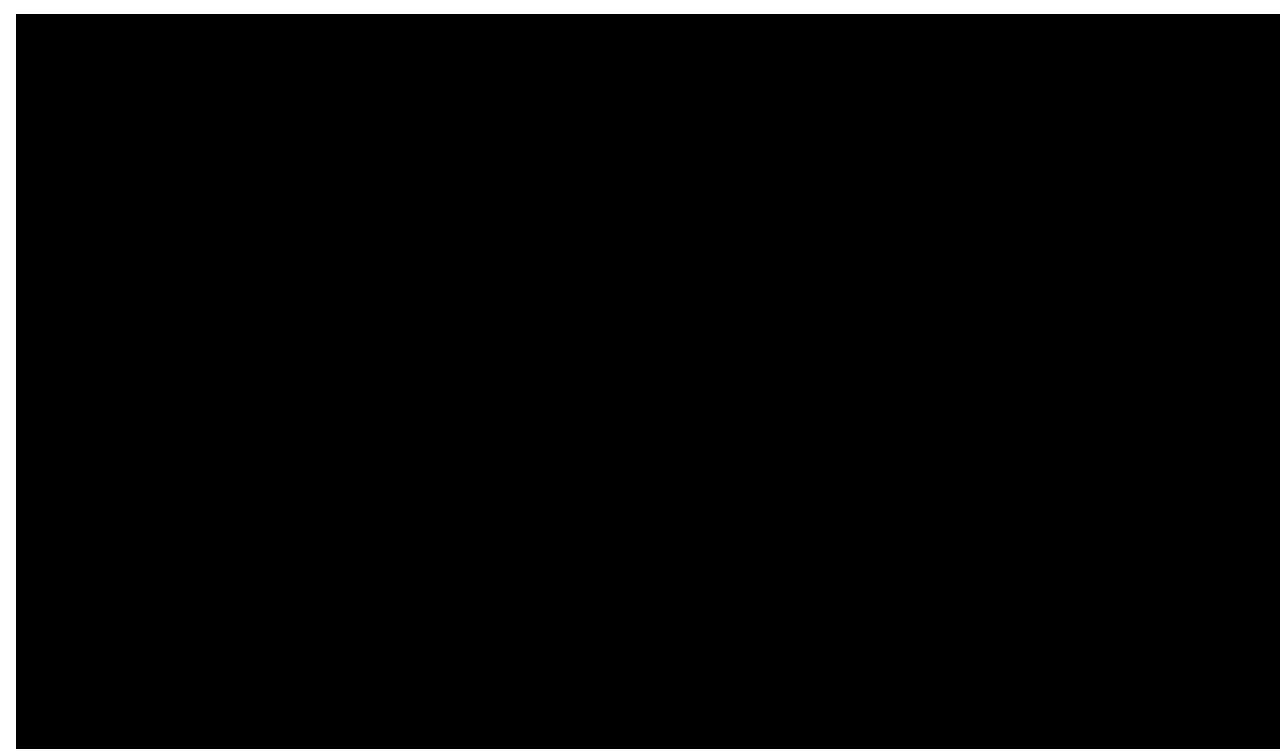

Tabla 7: Distribución de los programas más descargados desde Internet

T.u 
Igualmente, otros factores pueden ayudar a comprender el por qué de esta ausencia. Y es que la producción de ficción española se genera prácticamente en su totalidad desde productoras independientes y coproducciones. Esta práctica puede bien dificultar una gestión coordinada de las plataformas múltiples de distribución donde se alojan y distribuyen los contenidos. No solamente en el estricto sentido logístico de las acciones sino en la decisión de tener que abrir otras ventanas de difusión y gestionarlas externamente de forma eficiente. También puede obedecer al miedo que puede ocasionar el eventual preestreno vía Internet de un producto nacional que aún no se sabe cuánta audiencia lo irá a respaldar, como sucede con las miniseries y TV Movies. Consideramos que esta nueva vía, además de ofrecer múltiples facilidades al público: recepción activa, fragmentada, autónoma, acompañada de recursos multimedia de interacción y seguimiento de personajes; es una herramienta con enorme viabilidad comercial para el mercado consumidor español y para gestionar la promoción de las empresas realizadoras y sus producciones en el contexto internacional, porque abre enormemente los canales de difusión.

La necesidad de incidir en este nuevo sector, preferencial en el consumo y los hábitos del segmento juvenil, se ve reforzada por el incremento de la velocidad de descarga de archivos multimedia. A partir de ello se genera un aumento de las ventas y aumenta el uso de dispositivos ultra portátiles con gran capacidad de almacenamiento como Netbooks o Ipads, que interconectan el visionado de contenidos con las labores habituales del estudio, trabajo y la interacción intensiva con otros usuarios mediante Redes Sociales. Este cambio se ve reflejado en el aumento de la frecuencia de descargas de contenidos. Mientras las descargas diarias, semanales y mensuales aumentan entre 2008 y 2010 (ver Tabla 8), van disminuyendo las descargas con Poca Frecuencia y los que Nunca descargan contenidos. Podemos ver la rebaja de las dos últimas columnas. Por ejemplo, en 2008 era un 37\% el segmento que Nunca descargó contenidos, la cifra baja a un $32 \%$ en 2009 y a $28 \%$ en 2010. 


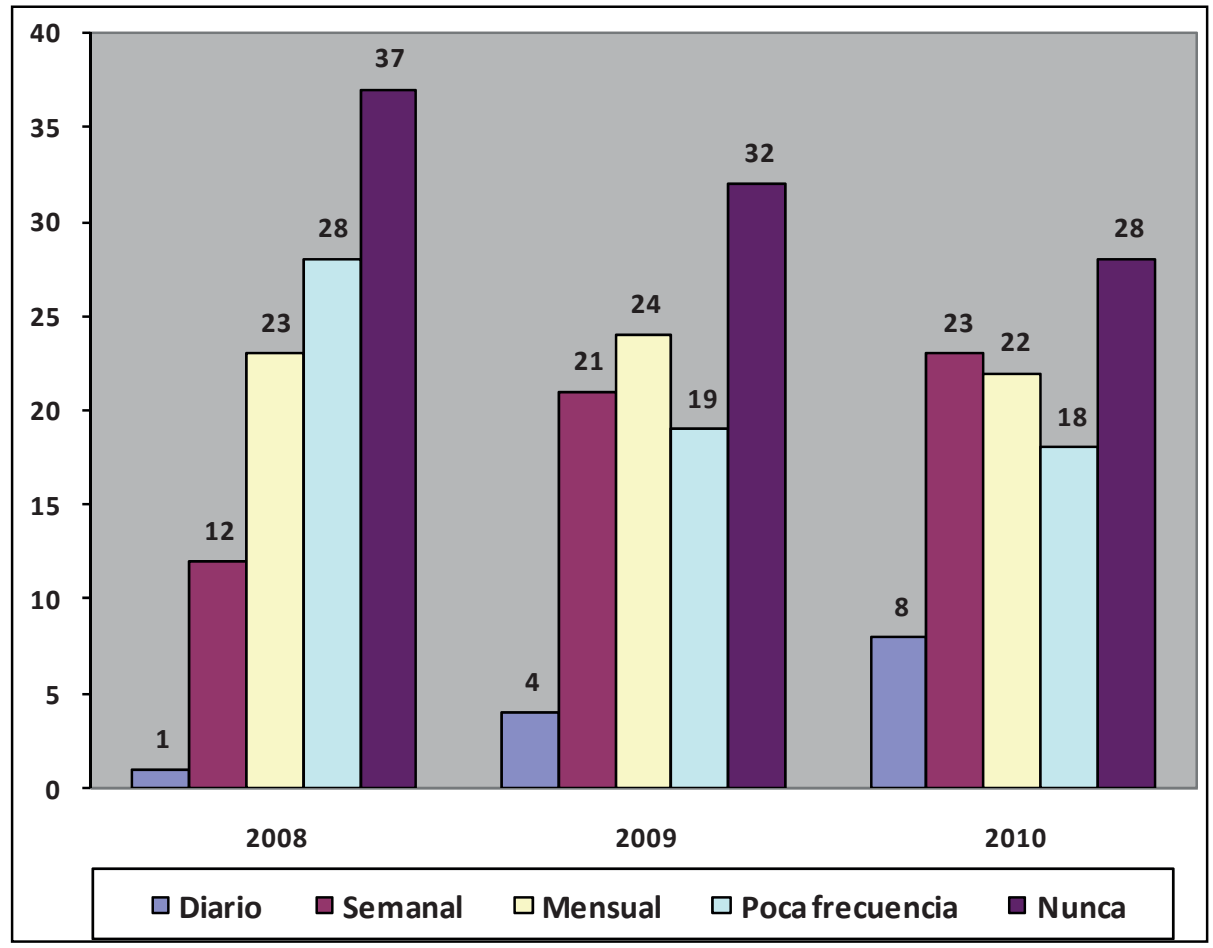

Tabla 8: Frecuencia de descargas de ficción desde Internet

Concretamente en 2010, según el último reporte de telefónica ${ }^{6}$, el tráfico global de vídeo por Internet ha crecido notablemente llegando a cuotas cercanas al 40\%. La cuota de visionado de TV por ordenador alcanza el 37\% y el intercambio de archivos llega a 40\% (ver Tabla 9). El video de Internet visto en la televisión aun es bajo, solo llega al 2,1\%, porque todavía no existe una homogenización de calidad de los archivos que incite verlos a través de pantallas de alta calidad que no harán más que destacar la baja resolución y las imperfecciones propias de los formatos de alta compresión. 


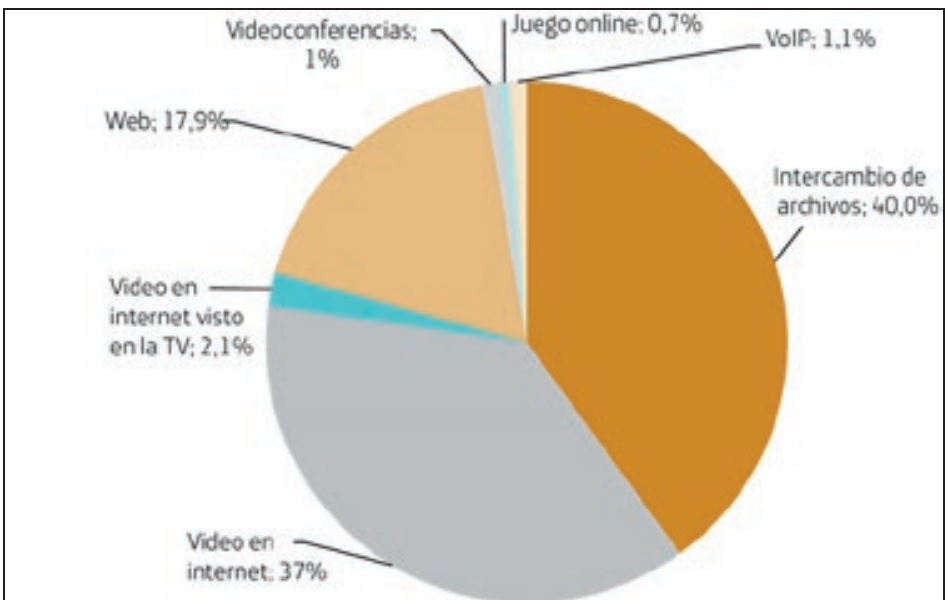

Tabla 9: Distribución del consumo de vídeo por Internet en el hogar

Fuente: La Sociedad de la Información en España 2010 - Fundación Telefónica

El caso tráfico en Internet móvil (ver Tabla 10) es incluso mayor llegando a cuotas del $51 \%$, falta saber con mayor exactitud en qué tipo de productos se concentra preferentemente el visionado. De todas maneras es un avance importante respecto del año pasado. Asimismo, el visionado on line a través de canales P2P se incrementa. Este dato de última hora, corrobora nuestros pronósticos acerca de un posicionamiento importante del uso de esta tecnología en el visionado televisivo.

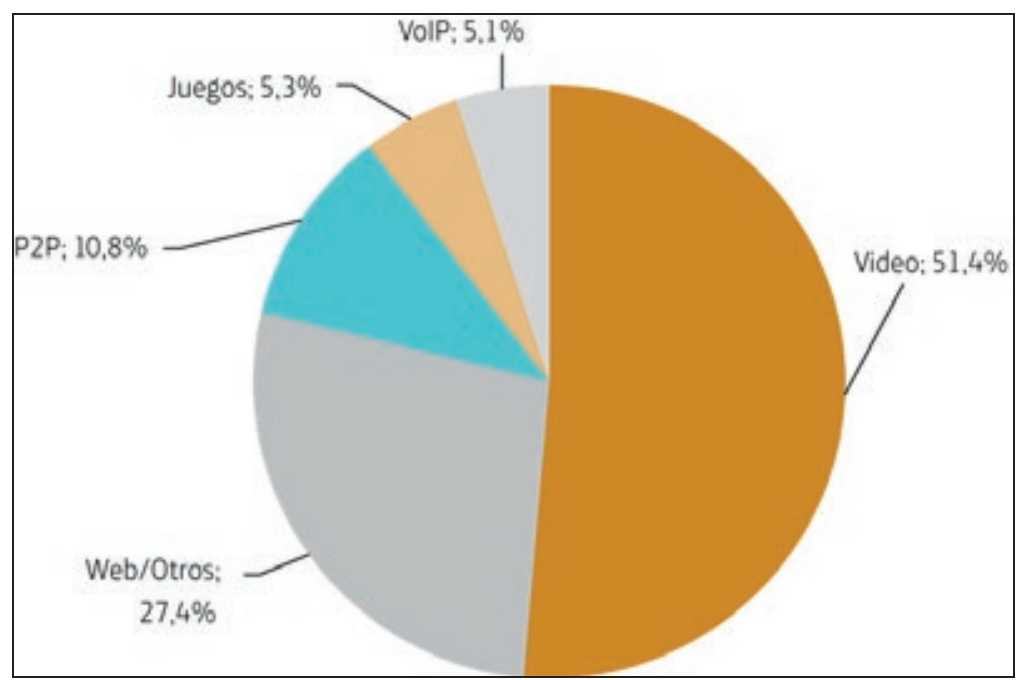

Tabla 10: Distribución del consumo de vídeo por Internet en teléfonos móviles Fuente: La Sociedad de la Información en España 2010 - Fundación Telefónica 


\section{DISCUSIÓN}

Tal y como hemos venido desarrollando nuestro análisis segmentado, discutiremos seguidamente los diferentes elementos que vienen incidiendo en la evolución y situación actual de la ficción audiovisual centrándonos alrededor de tres ámbitos: tecnológico, formas de consumo y finalmente, el contexto empresarial y las políticas públicas.

\subsection{La Tecnología}

En el sector audiovisual, el aporte tecnológico más importante se centra en el desarrollo de diversos dispositivos de transmisión y recepción de señales de video y televisión. A partir de la revisión actual dos ventajas cualitativas podemos percibir: la capacidad de prestaciones (resolución de pantallas, recepción multiformato, registro, memoria y transferencia de data) y, además, en el nivel flexibilidad para interactuar con diferentes fuentes propias empleadas en las rutinas prácticas de la producción audiovisual (otros equipos, marcas, modelos, sistemas operativos, etc.). En el sector profesional, actualmente conviven simultáneamente tres modelos de transmisión la TDT, HDTV y 3DTV, curiosamente con escazas formas de hacerse compatibles. Con un desarrollo y penetración desigual en cuanto a la implementación de plantas emisoras, adaptación de equipamiento en cadenas y productoras; así como en la adquisición de receptores compatibles de los usuarios. El cambio a la TDT, que aun continúa en proceso, apunta a un incremento de la oferta de canales pese a la implantación simultánea de emisiones en HD. La 3DTV de momento va un poco relegada y las experiencias acotadas se producen en el ámbito autonómico de Cataluña. Los televisores 3D, como siempre están en evolución pero aun no logran consolidarse como una alternativa seria. En esto tiene mucha influencia la nueva oferta de TV Web de Google TV y AppleTV. Esto parece indicar entonces, que los modelos digitales irán desvaneciéndose de la oferta y preferencias de los países y en cambio la IPTV, en base a la cual se configura el protocolo tanto de Google TV como de AppleTV, tomará la delantera del sector con la intención de convertirse en el modelo hegemónico. En esta línea, donde se aloja también la ficción, están los canales P2P. Funcionan bien desde servidores con señal en directo o 
como repositorio temático especializado, presentado en formato canal: EJ. Ustream, JustinTV, LiveTV, Freedocast; o mediante software: Sopcast, TVants, Stream Torrent, entre otros. Los móviles 4G, Ipads o Smartphones, por citar los más populares actualmente, ya están adaptados para recibir señal de televisión bajo cualquiera de estas posibles configuraciones y protocolos, solo falta resolver el acceso libre a la Red para que el consumo no esté restringido a una determinada zona de acceso WIFI.

\subsection{Consumo}

Los recientes estudios de audiencia confirman la diversificación del consumo y la consolidación del ordenador como pantalla sustituta preferencial para la recepción televisiva en general y ficcional en particular. La promoción de tarifas planas de alta velocidad y formas de acceso a la Red ha contribuido a mejorar las cuotas de consumo y a fomentar el visionado de vídeos y programas de TV in streaming o mediante descargas mediante hipervínculos. Por un lado las preferencias se orienta al uso de grandes servidores como Youtube, Google vídeos $\mathrm{u}$ otros alojamientos construidos para el visionado gratuito de películas o por pago como veocine.es, cinegratis.net, películas21.com. También se hace mediante las páginas web de las propias televisoras. En estas últimas actualmente encontramos una ingente cantidad de programas de ficción acompañados de todo un aparato de proyección colectiva: diseños persuasivos con animaciones, resúmenes, foros, materiales multimedia, RSS y enlaces directos para seguirlos en las principales redes sociales como Facebook, My Space o Twitter. La actualización y el feedback inmediato con actores y productores representan una ventaja importantísima en detrimento de la televisión convencional. Sin embargo, al parecer de momento, los productores nacionales, pese a sus esfuerzos evidentes, no son capaces de dominar el mercado de las descargas, aun cuando disponen de la empatía cultural de sus historias y audiencias. Curiosamente obtienen mejores resultados series extranjeras como House, CSI o Lost. 


\subsection{La dinámica empresarial y regulación del Estado}

El porcentaje elevado de presencia de productos ficcionales en las pantallas españolas constituye un primer dato valioso, considerando la caída general de la oferta, inversiones y publicidad por la crisis económica. Actualmente nos encontramos frente a un escenario dominado una vez más por las grandes canales nacionales, que se han visto fortalecidas por las fusiones impulsadas por el gobierno. La cadena de referencia para la emisión de ficción es Antena 3, le sigue Telecinco, TVE, La Sexta y Cuatro. Por su parte, los canales temáticos han mantenido con menos recursos el nivel y estilo de su programación, asegurando cuotas de audiencia bastante estimables. Pese a los esfuerzos por apoyar la producción propia y a través de ella proyectar su cultura e identidad, los canales gestionados por las comunidades autónomas, nuevamente han obtenido resultados positivos solo el contexto local. Se perciben grandes temores por trasuntar nuevos mercados dentro del propio país e internacionales.

Por el lado de las empresas productoras no se avistan mayores cambios. Los focos concéntricos siguen siendo entre unas pocas privilegiadas quienes negocian los mejores acuerdos para producir programas por encargo, adaptaciones o a medida de las necesidades e intuiciones de los directivos de las principales televisoras. Las coproducciones son mínimas por parte única de Globomedia, para solventar un proyecto con la televisora del País Vasco y una miniserie de dos capítulos solicitada expresamente por la cadena pública Televisión Española. Al margen de estas dos únicas experiencias, no se detectan proyectos innovadores en el mercado actual, aun cuando el trabajo de marketing vía web sí suponga variaciones y rompimientos de los estilos narrativos convencionales.

En esta falta de acción, buena parte de la responsabilidad recae en la actitud del Estado por no trazar una inevitable política regulatoria, pensada en ir más allá del simple incentivo a la fusión de los grandes conglomerados, asfixiados por las deudas a sus trabajadores y al propio Estado o apoyando a la industria desde su propio canal. Es incomprensible convivir con tres modelos de transmisión televisiva donde simplemente 
el libre mercado va ordenando y disponiendo cuál es el modelo a seguir hasta el momento que surja otro aparentemente superior. Sin mirar o adaptar siquiera las bondades de otros sistemas como el alemán o inglés. Eso, sin hablar nuevamente del consabido tema de los contenidos y su pertinencia en los horarios vespertinos. En esta vía, el papel de las productoras privadas puede desempeñar un rol sustancial para lograr dar un aporte a las cadenas al tiempo de asentarse como una industria fuerte y con posible alcance internacional. Para ello pueden imitarse los modelos exitosos de grandes empresas latinoamericanas como Televisa, Telemundo o Caracol, que curiosamente tienen el control de una franja importante del mercado nacional de ficción serial en formato telenovela.

Por otra parte, la revolución de Internet y las nuevas pantallas, pone sobre la mesa nuevos debates públicos devenidos del cambio de la lógica de circulación de programas; ante ellos las instancias competentes deben inevitablemente pronunciarse. Por Ej.: la regulación acerca de las formas de transmisión televisiva por IP y P2P plantea un debate permanente alrededor de los derechos de autor o la difusión internacional. Del mismo modo hace falta normar la legalidad de descargas y visionado para terceros usos. Impulsar el desarrollo de contenidos, y aplicaciones para facilitar la creación de productos y su descarga y reproducción a través de móviles y aparatos de reproducción portátil. Este campo es amplísimo y puede constituir la bisagra para unir tanto el trabajo de productores de diferentes plataformas como de centros de emisión y distribución de programas.

\section{CONCLUSIONES Y PERSPECTIVAS FUTURAS}

El presente artículo ha procurado construir una representación del estado actual del contexto audiovisual relacionado a la producción y negocio de la ficción en España. Se trata de una fotografía instantánea de la evolución de los sectores tecnológicos y económicos involucrados en el sostenimiento de una industria con características propias. Hemos podido constatar una falta de desarrollo y presencia mediática 
sostenida debido a la ausencia de un marco legal regulatorio y de promoción a la creación de contenidos para la televisión y los nuevos medios interactivos.

Primeramente, no queda otra explicación que el desarrollo de la gran tecnología de la comunicación: sistemas, dispositivos, formatos de registro, difusión y recepción de señales, es quien actualmente impone las prácticas, formas y estilos de consumo de las programaciones. Nunca antes se había visto una dependencia tan directa de los factores tecnológicos para el incremento de cualquier forma de experiencia mediática. Internet y la alta velocidad contribuyen de manera decisiva a este volcamiento de las audiencias que optan cada vez más por ver sus programas desde el ordenador o móviles 4G.

Es también la tecnología la que provoca la migración audiencias, en función de ello, creadores y anunciantes se concentran en aprovechar los espacios webs e interactivos para mejorar su contacto con los públicos cautivos. Consiguientemente, se produce una merma en la audiencia televisa, especialmente en el sector de los jóvenes nativo tecnológicos. En esta línea, parece ser que el avance generacional será el principal condicionante para le inmersión definitiva de los medios en los nuevos espacios. La televisión del hogar se convertirá muy pronto en una pantalla táctil, donde podrá accederse de forma instantánea a una multiplicidad de contenidos en grandes redes y repositorios interconectados. De cualquier manera, las experiencias actuales en el sector del cross media, son insuficientes y no trasvasan el aporte de una simple adaptación o cambio de soporte de difusión. Desde nuestro punto de vista, esta es la clave del desarrollo del modelo audiovisual, siempre y cuando satisfaga las demandas colectivas. En este sentido, las características naturales de los productos ficcionales son totalmente adaptables en su forma y estructura a las nuevas formas comunicativas de la portabilidad y la fragmentación, propias del entorno Internet. Las coproducciones siempre constituyen vías oportunas para darle multiperspectiva a nuevos proyectos los cuales deben contemplar la emisión multiplataforma. Son muy pocas las experiencias, pero las un sector Webseries pueden suministrar alternativas interesantes en cuanto a cuestiones creativas, narrativas y comerciales. 
De cara al futuro, falta potenciar la conexión entre fabricantes de dispositivos, distribuidoras y desarrolladores de contenidos para lograr que los productos se acomoden específicamente a las capacidades y bondades de los nuevos equipos. Debe procurarse una elección sensata de modelos de transmisión, considerando variables de ajuste propias del nuestro sistema político y social y no simplemente dejar que todo trasunte por la dinámicas del mercado. Esto no hace otra cosa que fortalecer cada día más a las grandes industrias, canales privados y debilitar la televisión pública. Muchos proyectos de ficción basados en recreaciones históricas no hubiesen podido salir en las pantallas si no hubiesen contado con el cofinanciamiento de las cadenas públicas. Se debe mantener la condición primigenia de Servicio Público de la televisión para cumplir con ciertos cánones que coadyuven a elevar la capacidad crítica y analítica de los mensajes. Esto, finalmente, es estricta responsabilidad del Estado a través de las instituciones reguladoras de ámbito nacional y/o autonómico y no hay otra alternativa vía que esperar la sensatez de sus decisiones en un tiempo indeterminado.

\section{REFERENCIAS}

- BIKFALVI, A, GARCÍA-REINOSO, VIDAL, I \& VALERA, F. (2009). A peer-to-peer IPTV service architecture for the IP multimedia subsystem in Int. J. Commun. Syst. 00:120

- BUSTAMANTE, E. (2008). Modelos internacionales de TDT: La Politica y los lobbies pueden frustrar las expectativas de diversidad. En Diálogos de Felafacs, No 77.

- CMT (2010). Informe anual, año 2010 de la Comisión del Mercado de las Telecomunicaciones

http://www.cmt.es/es/publicaciones/anexos/20100705_IA09_CMT_INFORME_A NUAL_2009_SENCER_BAIXA.pdf

- DELGADO, M. \& FERNÁNDEZ, D. (2007). La IPTV: Estructura de mercado y tipología de la oferta en España, en Zer 22, Universidad del País Basco.

- Impulsa TV (2010): Informe anual año 2009. Ministerio de Industria, Turismo y Comercio de España.

http:// www.televisiondigital.es/Terrestre/Paginas/Index.aspx

- ImpulsaTV (2010) Informe de Abril 2010 http://www.impulsatdt.es/pdf/boletin/INFORME_ABRIL_2010.pdf

- Ley 7/2009 de Medidas Urgentes en Materia de Telecomunicaciones http://www.mityc.es/telecomunicaciones/esES/Novedades/Paginas/RealDecretoLey1_2009.aspx 
- READING, Viviane (2008). Europe on the way to a high speed Internet economy. Brussels: Launch Press EITO. 